\title{
AN EXCEPTIONAL ARITHMETIC GROUP AND ITS EISENSTEIN SERIES
}

\author{
BY WALTER L. BAILY, JR. ${ }^{1}$
}

Communicated by Armand Borel, September 27, 1968

1. Introduction. Let $G_{R}$ be the simply-connected, real, Lie group of type $E_{7}$ which is isogenous to the full group of holomorphic automorphisms of a bounded symmetric domain in $\mathbf{C}^{27}$. It is the purpose of this note to announce results on a certain arithmetic subgroup $\Gamma$ of $G_{R}$ and its automorphic forms; in particular, we have proved that the automorphic forms for $\Gamma$ given by Eisenstein series have Fourier coefficients which are rational numbers with a certain Euler product expansion. Because the proofs are too long to give here, they will be presented elsewhere.

In this note, all our fields are of characteristic zero; we use $C, R$, $\boldsymbol{Q}$, and $\boldsymbol{Z}$ to denote respectively the complex numbers, the real numbers, the rational numbers, and the rational integers. If $V$ is an algebraic group, algebra, or vector space defined over $Q$, and if $k$ is a field containing $Q$, denote by $V_{k}$ the group of $k$-rational points of $V$. It is not necessary that the family of all the fields we consider, ordered by inclusion, have a maximal element.

2. Cayley numbers. We denote by $\sqrt{ }$ the ring of Cayley numbers constructed from the standard basis of eight units and multiplication table of [3]; this gives $\mathfrak{C}$ a $Q$-structure, and $\mathfrak{C}_{R}$ is a division algebra. The ring $\mathfrak{S}_{k}$ has an involution $a \rightarrow \bar{a}$, from which we define the trace function $T: a \rightarrow a+\bar{a}$, a bilinear form $B:(a, b) \rightarrow T(a \bar{b})$, and norm $N: a \rightarrow a \bar{a}$. We identify $k$ with the set of $a$ in $\mathfrak{S}_{k}$ such that $a=\bar{a}$.

Coxeter [3] has constructed a subring of $\mathfrak{E}_{R}$, which we denote by D, which is a lattice, contained in $\mathfrak{S}_{Q}$, of the real vector space $\mathfrak{S}_{R}$, such that $\mathrm{o} \cap \boldsymbol{R}=\boldsymbol{Z}$, and which has the further important properties: (1) $\mathrm{o}$ is self-dual with respect to $B($,$) ; (2) if a \in 0$, then $T(a)$ and $N(a)$ are integers; (3) $D$ is maximal with respect to the preceding properties; and (4) if $\beta_{1}, \cdots, \beta_{8}$ is a basis of $\mathrm{D}$ and if $a_{1}, \cdots, a_{8}$ are arbitrary elements of $\boldsymbol{Z}$, then there exists $a \in \mathcal{0}$ such that $B\left(\beta_{i}, a\right)=a_{i}, i=1$, $\cdots, 8$. We then have the

1 The author wishes to acknowledge support for research on the subject matter of this note from NSF grants GP 3903 and GP 6654, as well as from a senior post-doctoral NSF fellowship in the academic year 1965-1966. 
Lemma 1. Let $\mathfrak{o}_{1}=\{a \in \mathfrak{o} \mid N(a)=1\}$. If $x \in \mathfrak{C}_{R}$ satisfies $|B(x, c)| \leqq 1$ for all $c \in 0_{1}$, then $N(x) \leqq \frac{1}{2}$.

The above facts about $\mathfrak{C}_{R}$ and $\boldsymbol{D}$ are basic in proving the arithmetic part of our results.

3. Exceptional Jordan algebras. Let $\Im_{k}$ be the set of 3 by 3 matrices $X=\left(x_{i j}\right)$ over $\mathfrak{C}_{k}$ such that $x_{i j}=\bar{x}_{j i}$; in particular, $\xi_{i}=x_{i i} \in k$. This becomes a Jordan algebra, supplied with the product $X \circ Y$ $=\frac{1}{2}(X Y+Y X)$, where $X Y$ is the ordinary matrix product. One defines $\operatorname{tr}(X)=\sum_{i} x_{i i}$, an inner product $($,$) by (X, Y)=\operatorname{tr}(X \circ Y)$, and a symmetric trilinear form $(,, \quad)$ such that the associated cubic form $\operatorname{det} X$ has the formal appearance of a determinant [6]. Let $X$ be the bilinear mapping of $\Im_{k} \times \Im_{k}$ into $\Im_{k}$ defined by $(X \times Y, Z)$ $\equiv 3(X, Y, Z)$.

Let $K$ be the cone of squares of elements of $\Im_{R}$, and let $K^{+}$be its interior; if $X \in K^{+}$, then det $X \neq 0$. We define $\Im_{0}=\left\{X \in \Im_{R} \mid X=\left(x_{i j}\right)\right.$, $x_{i j} \in \mathcal{0}$ (in particular, $\left.\left.\xi_{i} \in Z\right)\right\}$. Then $\Im_{0}$ is a self-dual lattice with respect to $($,$) .$

4. $k$-forms of $E_{6}$ and $E_{7}$. Let $g_{k}=\left\{g \in G L\left(\Im_{k}\right) \mid \operatorname{det}(g X) \equiv \operatorname{det} X\right\}$ and let $g_{0}=\left\{g \in g_{Q} \mid g \Im_{0}=\Im_{0}\right\}$. One defines [7] an automorphism $g \rightarrow g^{*}$ of $g_{k}$ by $\left(g X, g^{*} Y\right) \equiv(X, Y)$. If $g \in g_{k}$, then $g(X \times Y)$ $=\left(g^{*} X\right) \times\left(g^{*} Y\right)$; moreover, $g_{0}$ is stable under that automorphism.

Using these facts, Lemma 1 , and [6, Theorem 12], one may prove

Proposition 1. The group $g_{R}$ is connected and $g_{0}$ is a maximal discrete subgroup of $\mathfrak{g}_{R}$. If two Q-parabolic subgroups of $\mathfrak{g}_{R}$ are conjugate, then they are conjugate by an element of $\mathcal{S}_{\mathbf{0}}$.

Let $X$ and $X^{\prime}$ be two copies of $\Im_{k}$, and let $\Xi$ and $\Xi^{\prime}$ be two copies of $k$. Define $W_{k}$ to be the direct sum of vector spaces, $X \oplus \boldsymbol{Z} \oplus X^{\prime} \oplus \mathbf{Z}^{\prime}$. If $w \in W_{k}$, we may write $w$ in terms of its components in the direct summands as $w=\left(X, \xi, X^{\prime}, \xi^{\prime}\right)$. Define [4] a quartic form $J$ on $W_{k}$ by $J(w)=\left(X \times X, X^{\prime} \times X^{\prime}\right)-\xi \operatorname{det} X-\xi^{\prime} \operatorname{det} X^{\prime}-\frac{1}{4}\left(\left(X, X^{\prime}\right)-\xi \xi^{\prime}\right)^{2}$ and a skew-symmetric bilinear form $\{$,$\} on W_{k} \times W_{k}$ by

$$
\left\{w_{1}, w_{2}\right\}=\left(X_{1}, X_{2}^{\prime}\right)-\left(X_{2}, X_{1}^{\prime}\right)+\xi_{1} \xi_{2}^{\prime}-\xi_{2} \xi_{1}^{\prime},
$$

where $w_{i}=\left(X_{i}, \xi_{i}, X_{i}^{\prime}, \xi_{i}{ }^{\prime}\right)$. Define $G_{k}$ to be the group of all linear transformations of $W_{k}$ leaving $J$ and $\{, \quad\}$ invariant. Let $W_{0}$ be the lattice of all $w \in W_{Q}$ such that $X, X^{\prime} \in \mathcal{Y}_{0}$, and $\xi, \xi^{\prime} \in Z$, and define

$$
\Gamma=G_{0}=\left\{g \in G_{Q} \mid g W_{0}=W_{0}\right\} .
$$

Proposition 2. The group $G_{R}$ is connected and $\Gamma$ is a maximal dis- 
screte subgroup of $G_{R}$. If two Q-parabolic subgroups of $G_{R}$ are conjugate, then they are conjugate by an element of $\Gamma$.

5. The symmetric domain. It is known [4] that $G_{R}$ is that real, connected, and simply-connected form of $E_{7}$ which is isogenous to the holomorphic automorphism group of a noncompact symmetric, hermitian space $D$ of 27 dimensions. The space $D$, which is complex analytically isomorphic to a bounded domain in $C^{27}$, may also be identified with the tube domain [5]

$$
\mathfrak{J}=\left\{Z=X+i Y \in C^{27} \mid Y \in K^{+}\right\},
$$

where $K^{+}$is defined as in $\S 3$. The group $G_{R}$ contains a subgroup $P^{+}$ which, via the action of $G_{R}$ on $\mathrm{J}$, is isomorphic to the group of all translations: $Z \rightarrow Z+A, A \in \mathfrak{\Im}_{R}$; it also contains the inversion $\iota: Z \rightarrow-Z^{-1}$ (Jordan algebra inverse); and $\iota$ and the group $P^{+}$generate $G_{R}$. Moreover, $G_{R}$ contains a subgroup isomorphic to $g_{R}$; if $g \in g_{R}$, then the action of $g$ on $J$ is the complexification of its action on $\Im_{R}$. There is a unique parabolic subgroup $\mathbb{P}$ of $G_{R}$ containing $g_{R}$ and $P^{+}$. Let $P_{0}^{+}=P^{+} \cap \Gamma$, identify $g_{0}$ with $\mathscr{g}_{R} \cap \Gamma$, and let $\Gamma_{0}=\Gamma \cap \rho$.

6. Automorphic forms. If $g \in G_{R}$, denote by $j(Z, g)$ the functional (jacobian) determinant of $g$ at $Z$ (we view $g$ as acting on the right). We remark that $j(Z, \imath)=(\operatorname{det} Z)^{-18}$, as follows from an easy calculation.

A holomorphic function $f$ on $\mathfrak{I}$ which satisfies the identity

$$
j(Z, g)^{m} f(Z \cdot g)=f(Z), \quad g \in \Gamma, Z \in \mathcal{J},
$$

where $m$ is an even integer $\geqq 0$, is called an automorphic form of weight $m$ for $\Gamma$. The relation (1) implies that $f$ is invariant under $\Gamma_{0}$, and in particular $f(Z+A)=f(Z)$ for all $A \in \Im_{\text {o }}$. Hence $f$ has a Fourier expansion

$$
f(Z)=\sum_{r} a(T) \epsilon((T, Z)),
$$

where the sum is over $T \in \Im_{0}$ and $\epsilon(x)=e^{2 \pi i x}$; by well-known principles $[2, \$ 10.14]$, one may even assert that $a(T)=0$ if $T \notin K$.

7. Eisenstein series. If $m$ is a positive even integer, we define

$$
E_{m}(Z)=\sum_{\gamma \in \Gamma / \Gamma_{0} j(Z, \gamma)^{m}, \quad Z \in J .}
$$

This series converges uniformly on compact subsets' of $J[2, \S 7.2]$ and is an automorphic form of weight $m$ for $\Gamma$. Hence it has a Fourier expansion

$$
E_{m}(Z)=\sum_{T \in \Im_{\text {on }}} a_{m}(T) \epsilon((T, Z)) .
$$

One may prove, using in part some ideas of Siegel $[8, \S 5]$, that the 
automorphic forms $E_{m}$ generate the field of automorphic functions for $\Gamma$. It follows without great difficulty that if $k$ is a field containing all the Fourier coefficients $a_{m}(T)$ for all $m$ and $T$, then $k$ is a field of definition for the Satake compactification [2, pp. 482-485] of $J / \Gamma$, viewed as a projective variety.

\section{The Fourier coefficients. One may prove}

THEOREM. All of the Fourier coefficients $a_{m}(T)$ are rational numbers.

We indicate very briefly how this may be proved for the case $T \in K^{+}$(so that det $T \neq 0$ ). The general case is similar.

Denote by $Q_{p}$ the $p$-adic completion of $Q$, and by $Z_{p}$, the ring of $p$-adic integers, for any rational prime $p$. Let $\Im_{0_{p}}=\Im_{0} \otimes_{Z} Z_{p}$, and let $\mathscr{I}_{0_{p}}$ be the stabilizer in $\mathscr{S}_{Q_{p}}$ of $\mathfrak{S}_{O_{p}}$.

LEMMA 2. If $u \in \Im_{Q_{p}}$, then there exists $g \in \mathscr{I}_{\mathfrak{o}_{p}}$ such that $g \cdot u=\left(u_{i j}^{\prime}\right)$ satisfies $u_{i j}^{\prime}=0$ if $i \neq j$.

(This is true for every rational prime $p$, including 2.)

Definition. Let $u \in \Im_{Q_{p}}$. If $g \in \mathcal{G}_{0_{p}}$ is as in Lemma 2 , let $\kappa_{p}(u)$ denote the archimedean absolute value of the product of the reduced denominators of the nonzero elements of $g \cdot u$ (so that $\kappa_{p}(u)$ is a nonnegative power of p). Let $\kappa(u)=\prod_{p} \kappa_{p}(u)$, where the product is over all rational primes.

Using the Poisson summation formula, one may prove, as in [8, §7], that if $Z \in J$, then for any even positive $m$,

(3) $\sum_{\lambda \in \mathfrak{Y}_{0}} \operatorname{det}(Z+\lambda)^{-18 m}=\Delta(m) \cdot \sum_{T \in \mathfrak{S}_{0} \mathfrak{I}_{0}} \cap K^{+} \operatorname{det} T^{18 m-9} \epsilon((T, Z))$,

where

$$
\Delta(m)=2^{54 m} \cdot \pi^{54 m-12} \cdot \prod_{n=0}^{2} \gamma(m-4 n)^{-1},
$$

$\gamma$ being the gamma function. Using (3) to transform the series for $E_{m}$, one obtains, analogously to $[8, \S 7]$,

$$
a_{m}(T)=\Delta(m) \cdot \operatorname{det} T^{18 m-9} \cdot \sum_{u \in \mathfrak{S}_{Q} \bmod \Im_{0}} \epsilon((T, u))_{\kappa}(u)^{-18 m},
$$

and the sum on the right is equal to $\prod_{p} S_{p}$, with

$$
S_{p}=\sum_{u \in \Im_{Q_{p}} \bmod \Im_{0_{p}}} \epsilon_{p}\left((T, u) \kappa_{p}(u)^{-18 m},\right.
$$

where $\epsilon_{p}$ is a character on $\boldsymbol{Q}_{p} / \boldsymbol{Z}_{p}$ which is not trivial on $p^{-1} \boldsymbol{Z}_{p}$. Then, using a generalization of Hensel's lemma and some related ideas [1], one sees that for each $p$, the expression defining $S_{p}$ is a sum of terms, 
each of which is itself a (finite) sum over an orbit of $\mathscr{S}_{0_{p}}$ in $\Im_{Q_{p}}$ modulo $\Im_{o_{p}}$; those terms are zero except for a finite number of orbits of $\Im_{0_{p}}$, and for almost all $p$, one need only consider the sum over $u$ such that $p u \in \Im_{o_{p}}$. From the first fact, one sees easily that each $S_{p}$ is a rational number, and by the second fact, the calculation of $S_{p}$ is reduced, for all but a finite number of $p$, to an enumerative calculation based on results of [7] and on elementary properties of character sums (in a way entirely different from the procedure in $[8, \S 7])$. The result is that for all but a finite number of $p$, we have

$$
S_{p}=\left(1-p^{-18 m}\right)\left(1-p^{4-18 m}\right)\left(1-p^{8-18 m}\right) .
$$

Combining (4), (5), and (6), one obtains the result that $a_{m}(T)$ is a rational number. As we have said, our method of evaluating the series $S_{p}$ is quite different from that of [8] for evaluating series analogous to $S_{p}$ in a classical case. In [8], use is made of Gaussian sums and their relation to the interpretation of $S_{p}$ as a representation density for quadratic forms. The absence, until now at least, of an analogous explanation in this exceptional case was what forced us to find another way of evaluating $S_{p}$. A problem of interest that remains is to find, if possible, an algebraico-geometric interpretation of the Euler factors (6), similar to the classical expression (in other cases) of such factors as representation densities of one quadratic form by another.

\section{REFERENCES}

1. W. L. Baily, Jr., On Hensel's lemma and exponential sums (to appear).

2. W. L. Baily, Jr. and A. Borel, Compactification of arithmetic quotients of bounded symmetric domains, Ann. of Math. (2) 84 (1966), 442-528.

3. H. S. M. Coxeter, Integral Cayley numbers, Duke Math. J. 13 (1946), 561-578.

4. H. Freudenthal, Beziehungen der $E_{1}$ und $\mathrm{E}_{\Varangle}$ zur Oktavenebene. I, Nederl Akad. Wetensch, Proc. Ser. A. 57 (1954), 218-230.

5. U. Hirzebruch, Über Jordan-Algebren und beschränkte symmetrische Gebiete, Math. Z. 94 (1966), 387-390.

6. N. Jacobson, Some groups of transformations defined by Jordan algebras. III, J. Reine Angew. Math. 207 (1961), 61-85.

7. J. G. M. Mars, Les nombres de Tamagawa de certains groupes exceptionnels, Bull. Soc. Math. France 94 (1966), 97-140.

8. C. L. Siegel, Einfiihrung in die Theorie der Modulfunktionen n-ten Grades, Math. Ann. 116 (1939), 617-657.

University of Chicago, Chicago, Illinois 60637 